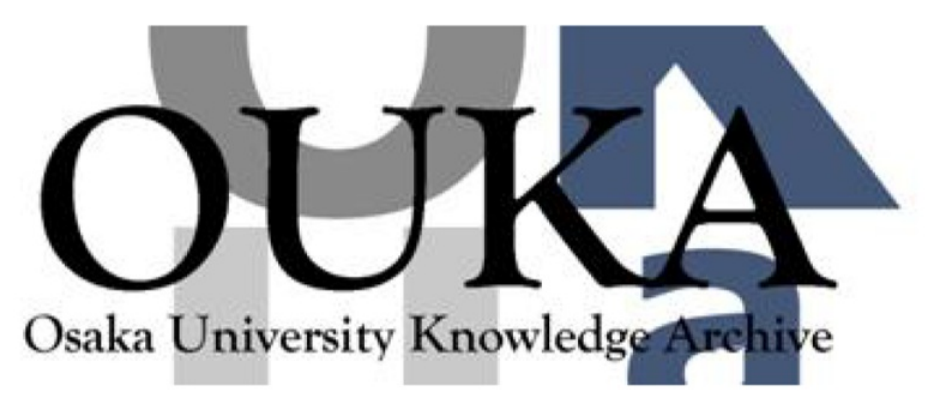

\begin{tabular}{|c|l|}
\hline Title & $\begin{array}{l}\text { Active liquid-crystal deflector and lens with } \\
\text { Fresnel structure }\end{array}$ \\
\hline Author(s) & $\begin{array}{l}\text { Shibuya, Gi ichi; Yamano, Shohei; Yoshida, } \\
\text { Hiroyuki et al. }\end{array}$ \\
\hline Citation & $\begin{array}{l}\text { Proceedings of SPIE - The International Society } \\
\text { for Optical Eng ineering. 10125 p. 101250V }\end{array}$ \\
\hline Issue Date & $2017-02-15$ \\
\hline oaire:version & VoR \\
\hline URL & https://hdl. handle. net/11094/76957 \\
\hline rights & \\
\hline Note & \\
\hline
\end{tabular}

Osaka University Knowledge Archive : OUKA

https://ir. Library. osaka-u. ac. jp/

Osaka University 


\title{
Active liquid-crystal deflector and lens with Fresnel structure
}

\author{
Giichi Shibuya, Shohei Yamano, Hiroyuki Yoshida, Masanori Ozaki \\ Osaka Univ., 2-1 Yamadaoka, Suita, Osaka 565-0871, Japan
}

\begin{abstract}
A new type of tunable Fresnel deflector and lens composed of liquid crystal was developed. Combined structure of multiple interdigitated electrodes and the high-resistivity (HR) layer implements the saw-tooth distribution of electrical potential with only the planar surfaces of the transparent substrates. According to the numerical calculation and design, experimental devices were manufactured with the liquid crystal (LC) material sealed into the sandwiched flat glass plates of $0.7 \mathrm{~mm}$ thickness with rubbed alignment layers set to an anti-parallel configuration. Fabricated beam deflector with no moving parts shows the maximum tilt angle of $\pm 1.3 \mathrm{deg}$ which can apply for optical image stabilizer (OIS) of micro camera. We also discussed and verified their lens characteristics to be extended more advanced applications. Transparent interdigitated electrodes were concentrically aligned on the lens aperture with the insulator gaps under their boundary area. The diameter of the lens aperture was $30 \mathrm{~mm}$ and the total number of Fresnel zone was 100 . Phase retardation of the beam wavefront irradiated from the LC lens device can be evaluated by polarizing microscope images with a monochromatic filter. Radial positions of each observed fringe are plotted and fitted with $2^{\text {nd }}$ degree polynomial approximation. The number of appeared fringes is over 600 in whole lens aperture area and the correlation coefficients of all approximations are over 0.993 that seems enough ideal optical wavefront. The obtained maximum lens powers from the approximations are about $\pm 4 \mathrm{~m}^{-1}$ which was satisfied both convex and concave lens characteristics; and their practical use for the tunable lens grade eyeglasses became more prospective.
\end{abstract}

Keywords: Liquid-Crystal devices, Fresnel, Electro-Optical devices, deflector, tunable

\section{INTRODUCTION}

Liquid crystal (LC) lenses ${ }^{[1]-[5]}$ and deflectors are actively studied in prospect of developing smart optical devices with tunability. In particular, LC lenses enable tuning of the lens power and polarity, and LC deflectors can change the deflection angle with both directions. However, the tunable range of the conventional devices were not so large that their applications were rather limited. Therefore, we have applied the Fresnel structure to expand the aperture of the lens and also the deflection angle with current thickness of the LC layer. Combined structure of multiple interdigitated electrodes and a high-resistivity (HR) layer induces a saw-tooth distribution of electrical potential under the planar transparent substrates ${ }^{[6]}$. The optimal frequency of the driving voltage was found to depend on the resistivity of the HR layer, the pitch of the interdigitated electrode and the structure of the thin films. The deflection angle of the beam incident normally on the device increased continuously with an increase in the applied voltage. Because of the Fresnel structure in which the wavefront is made periodic in small segments, a larger maximum deflection angle was achieved compared to the device without the Fresnel structure. However, when the segment pitch becomes smaller, the influence of diffraction light gets larger and they sometimes mixed with the deflected main beams. Therefore two kinds of incident beams were compared with the deflected light image on the screen. Captured image through the LC deflector and micro camera was also observed. When the different voltage was applied on the interdigitated electrodes of the LC device, ISO12233 test pattern image through the camera was vibrated and that can be apply for the OIS without any actuators. The haze of the image was found to be appeared when the irradiated light was deflected. What makes the images to be hazy and how to suppress the haze was also discussed.

We also prepared LC lens samples with a larger diameter of $30 \mathrm{~mm}$. Extraction electrodes arranged to cross over the circular interdigitated electrodes was patterned on the same surface. When we place the insulator gap structure around them, obtained interference fringes had less distortion and found to be contributed to decrease the manufacturing cost. Lens power defined by the reciprocal of the focal length was estimated from the obtained interference fringes. Calculated maximum lens powers were $+3.97 \sim-2.25 \mathrm{~m}^{-1}$ that shows difference with the polarity of the lens. During the 
investigation of the asymmetric characteristics, optimal driving frequency was found to be varied from the circular segment pitch on the lens pattern and how to decrease the dependence of that was also discussed.

\section{SIMULATION AND SAMPLE MANUFACTURING}

Figure 1 shows the device structure and calculated potential on the top of the LC layer. Optimal dimensions of the interdigitated electrodes and the parameters of thin film layers were determined from a finite element analysis, and experimental sample cells were fabricated ${ }^{[6]}$. LC material was sandwiched by glass plates of $0.7 \mathrm{~mm}$ thickness. Interdigitated electrodes, insulator, HR film were sequentially deposited on the same surface of the upper glass. Interdigitated electrode was patterned from the transparent indium-tin oxide (ITO) film on the glass. Used LC material was a nematic and polyimide layers were formed on both substrates for planar alignment of the LC; the polyimide layer was rubbed along the diagonal of the square-shaped substrates, and the two substrates were set to an "anti-parallel" configuration in the cell. Measure key point of the LC device process is the microstructure of the transparent electrodes. Patterning, etching and lift-off were also taken into the process to form the fine pattern of interdigitated electrodes and the insulator gap on the HR layer. Manufacturing pattern seems similar to the semiconductor process but not so hard to perform because the dimension of the LC device is not so fine, we suppose.
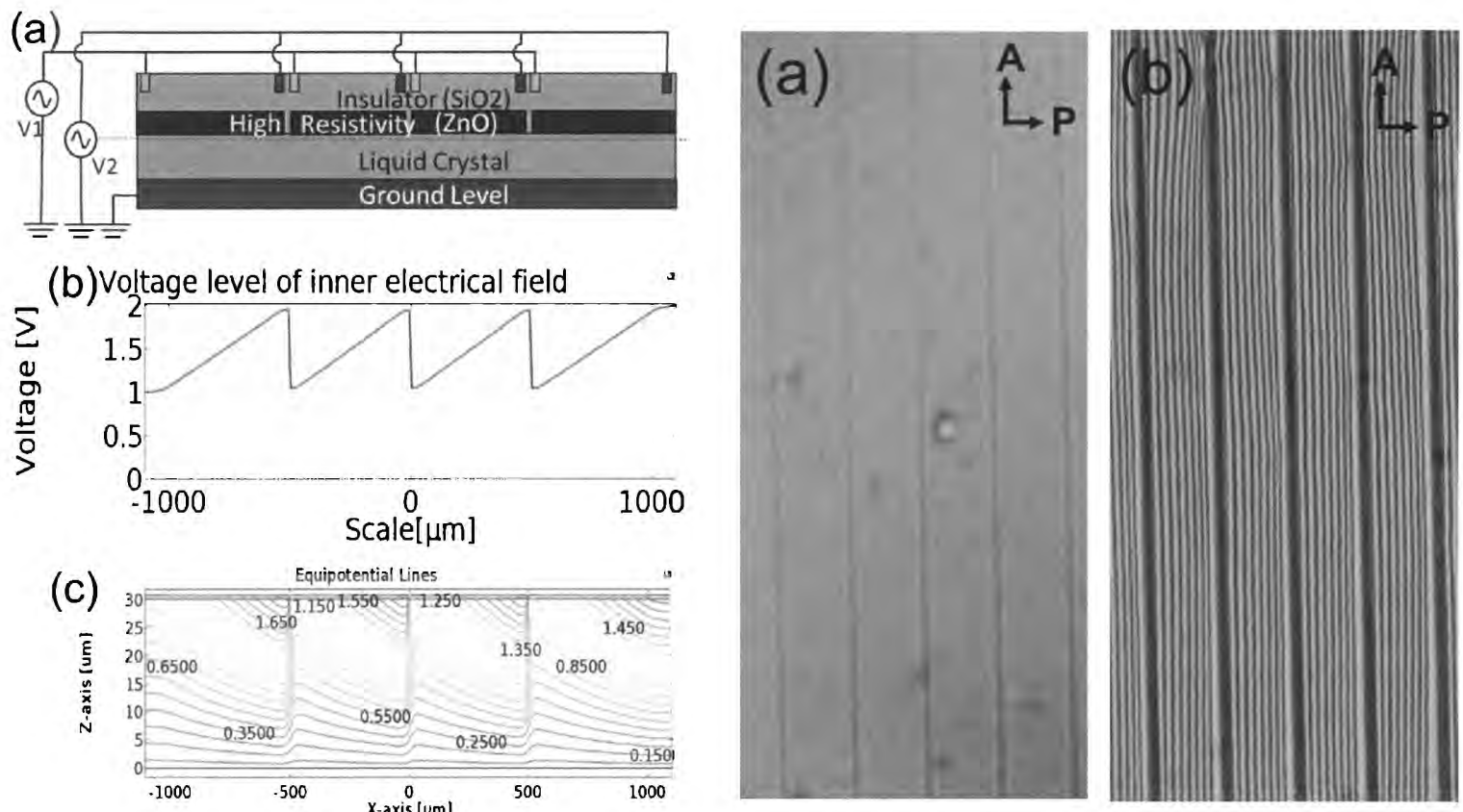

Figure 1. Simulation model and result of LC deflector.

(a) A cross-section of LC deflector device structure.

(b) Calculated potential on the top of LC layer.

(c) Contour of potential in the LC layer.

Figure 2. Optical micrograph of LC deflector device.

(a) Device with no voltage application.

(b) Device with voltage application.

Figure 2 (a) shows the optical micrograph of manufactured deflector device with no voltage application. The width and the space of the interdigitated electrode were set to 20 and $10 \mu \mathrm{m}$, respectively. The construction pitch of the periodic pattern of the segment was set to $200 \mu \mathrm{m}$. Figure 2 (b) shows the same device under the application of two different voltages, $\left(V_{1}, V_{2}\right)$ of $(1.9,0.35) \mathrm{V}_{\mathrm{mms}}$ to the each electrode as shown in Fig.1 (a). Periodic interference pattern was observed upon the applying electric field. Interference fringes with equal intervals indicates the saw-tooth refractive 
index occurred from the distribution of the electrical potential which is the effect of the high resistivity layer ${ }^{[7]}$. The gap width of the HR layer was set to $20 \mu \mathrm{m}$, which was wider than that of the interdigitated pattern to keep the alignment margin of the manufacturing process.

\section{LC DEFLECTOR}

LC deflector can make the incident light beam incline with the linear sloped distribution of refractive index. When the optical deflector will be applied for the OIS, over 3 times of larger deflection angle than current one is required. However, the birefringence value of the LC, $\Delta n$; a measure factor of their performance is rather limited, hence, we have to obtain the larger retardation only by increasing the thickness of the LC layer with the suffer of switching speed loss or the appearance of physical disclination. In this case, Fresnel structure is also effective solution which has the segmented structure in order to decrease the thickness of the optical material. Deflection angle can be enlarged with increasing of the number of the segment. Then, we have evaluated the manufactured samples of LC deflector to discuss their characteristics and applications.

\subsection{Deflection Angle}

The tilt angle of the incident beam was measured by the movement of the image of irradiated beam on the screen placed at a distance of $1 \mathrm{~m}$ from the optical deflector device. Beam spot image shifted in the direction perpendicular to the interdigitated electrodes as the voltage was applied. Obtained maximum tilt angles of the deflectors were $\pm 0.8 \mathrm{deg}$. For the device with $0.333 \mathrm{~mm}$ pitch and $\pm 1.3 \mathrm{deg}$. for the $0.2 \mathrm{~mm}$ pitch device. The observed thickness of the LC cell gap was $30.5 \mu \mathrm{m}$ and $27.6 \mu \mathrm{m}$, respectively, which yields a theoretical maximum retardation $\Delta n d$ of $5.67 \mu \mathrm{m}$ and tilt angle of $\tan ^{-1}(5.67 / 333)=0.975 \mathrm{deg}$. in the former case, and the retardation was $5.13 \mu \mathrm{m}$ and the tilt angle was $\tan ^{-}$ ${ }^{1}(5.13 / 200)=1.47 \mathrm{deg}$. in the latter case. Compared with these values, the obtained results from the measuring seem to be reasonable and the effect of the optical deflection with the Fresnel type of the LC device was clarified.

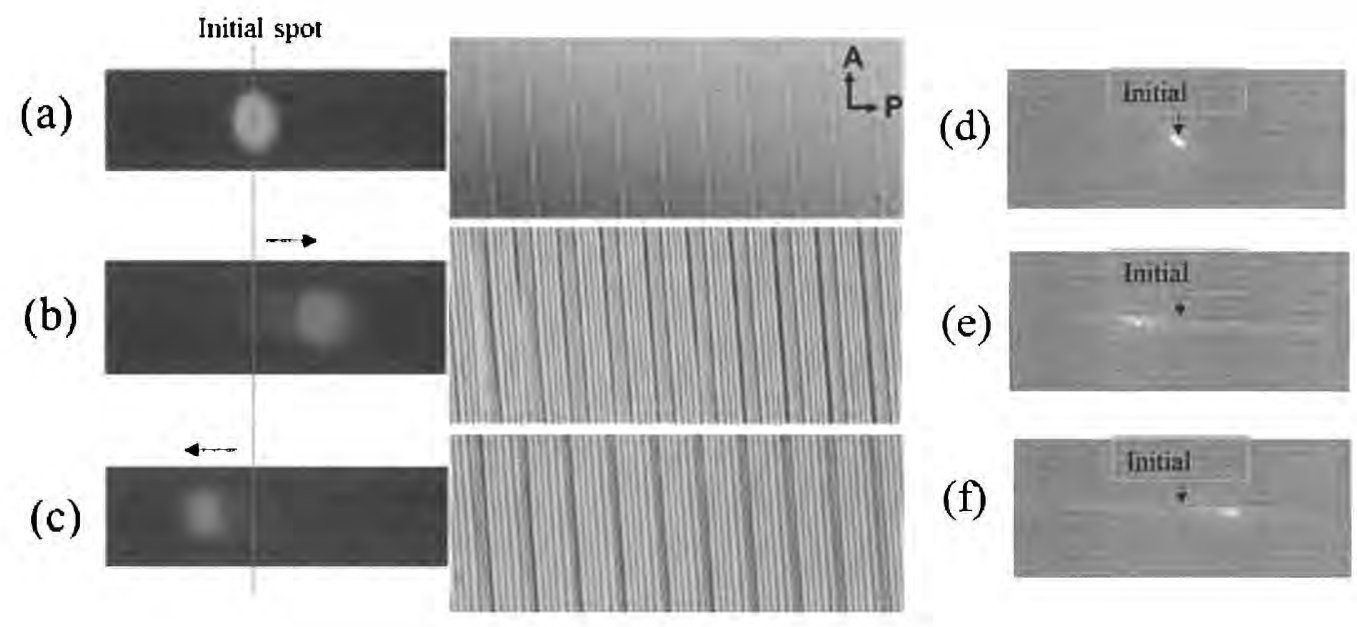

Figure 3. Observed beam spots on screen graph papers and interference fringes observed under each voltage application. Applied voltages $\left(V_{l}, V_{2}\right)$ [ $\left.\mathrm{V}_{\mathrm{rms}}\right]$ are as follows; (a),(d), $(0.7,0.7),(\mathrm{b}),(\mathrm{e}),(1.4,0.7),(\mathrm{c}),(\mathrm{f}),(0.7,1.4)$

Figures of (d) (f) are the comparison of the spot images whose irradiated beams are from laser diode.

Figure 3 shows the spot images of the irradiated beams on the screen paper under the voltage application and observed interference fringe patterns under the same driving conditions. White LED beam was used as the light source. Sample cell was set with their longitudinal line of the interdigitated electrodes to the vertical. Analyzer was set after the deflector device with the polarization plane parallel to the rubbing direction of the LC deflector cell. Wavelength of the used band 
pass filter on the microscope was $568 \mathrm{~nm}$. Frequency of the applied voltage was set to $1 \mathrm{kHz}$. Figure 3 (a) shows the spot image with the applied voltages $\left(V_{1}, V_{2}\right)$ of $(0.7,0.7) \quad \mathrm{V}_{\text {rms }}$, respectively. This voltage is less than the threshold level of the LC material and all the incident beam goes straight to the central spot. In this case, no interference fringes were observed under the microscope, as shown in the picture on the right side. Figure 3 (b) shows the spot image with the applied voltages $\left(V_{I}, V_{2}\right)$ of $(1.4,0.7) \mathrm{V}_{\mathrm{rms}}$, respectively. In this case, the number of observed interference fringes is 5 in each segment. The incident beam was deflected to the right, and the spot appeared at a distance of $17 \mathrm{~mm}$ from the initial position. When the applied voltages $\left(V_{1}, V_{2}\right)$ were interchanged into $(0.7,1.4) \mathrm{V}_{\mathrm{mms}}$, the obtained deflection angle was almost the same except that its direction was changed to the opposite side as shown in Fig.3 (c). As described in the pictures, deflected beam spots have a little residual image from the non-deflected previous spot. This intensity of residual image was found to be changed with the shift of beam irradiation area in the LC cell. Therefore, nonhomogeneousness of the sample cell seems to be one of the root cause of the residual image. However, some amount of the diffraction light from the periodic pattern of the interdigitated segments will be actually included. LC optical deflector comprised with the diffractive grating was studied ${ }^{[8]-[10]}$, and similar saw-tooth distribution was also applied due to increase the diffraction efficiency [9][10]. On the other hand, liquid crystal device with micro prism arrays and the transparent electrodes, shows the high deflection angle of over 10 degrees with the optical refraction ${ }^{[10]}$. Figure 3 (d) (f) shows the comparison of the spot images whose irradiated beams are from laser diode which has higher coherency than white light. More diffraction component of the irradiated beam was observed at the side of the main beam. However, this LC deflector is not a diffractive device, so that no diffraction beam should desirable to be irradiated no matter whether the light source is white or monochromatic. Hence, our necessary challenge is the reduction of the diffraction beam with the effective design of the periodic electrode and the advanced control of the driving voltage. Another approach is that we have to find out the limit intensity of the diffraction beam for each purposes and establish the optimal application and the driving method for the new LC device.
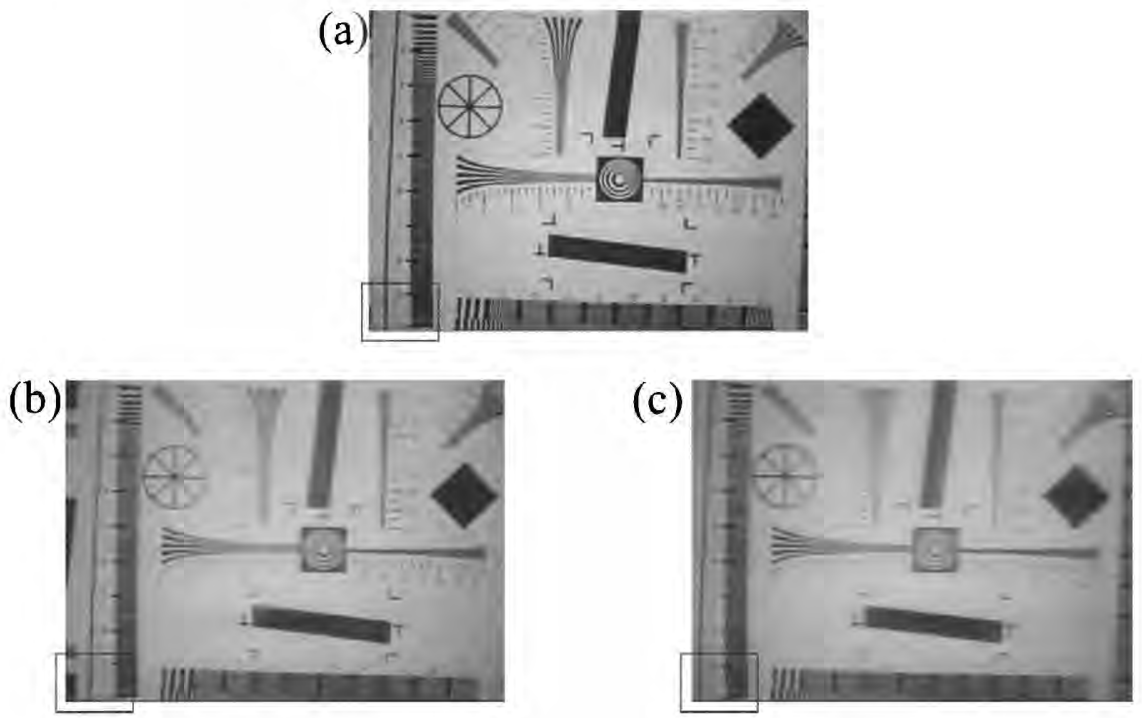

Figure 4. Captured images of the ISO12233 test pattern with the deflection light through the LC deflector. Applied voltages $\left(V_{l}, V_{2}\right)\left[\mathrm{V}_{\text {rms }}\right]$ are as follows; (a), $(0.35,0.35),(\mathrm{b}),(1.41,0.35),(\mathrm{c}),(0.35,1.41)$

\subsection{Image through the LC deflector}

In order to discuss the application for OIS function, we tried to evaluate the image through the LC deflector. Figure 4 shows the captured images of the ISO12233 test pattern with the deflection light through the LC deflector with the micro camera (ELECOM Inc., UCAM-DLI500TN, 5M pixels, C-MOS 1/4 inch,). Captured image was vibrated horizontally as shown in the blue square area at the bottom left corners of the pictures by voltage application of described each values. However, images with the deflected light includes haze in all area as described on Fig.4 (b), (c). In particular, part of the 
images seem to be duplicated on Fig.4 (c). Haze level was evaluated by the modulation ratio from the object image ${ }^{[1]}$ and found to be larger with the decreasing of the segment pitch.

Therefore, we suppose that this haze occurs from the narrow plateau area of saw-tooth potential distribution caused by the patterned transparent electrode. The incident light through plateau area goes straight and doesn't deflect at all. This components of light will be supposed to be mixed to the deflected light and make increase of the haze on the captured image. According to this assumption, when the segment pitch is designed to be smaller, the interdigitated electrode and the gap width should be designed also smaller to decrease the dimensional ratio of plateau area of the saw-tooth distribution of the wavefront. To distinguish which is more dominant factor the straight light, scattered light or the diffracted light is also important. When we apply the deflector for the use of correction for captured image like OIS, this haze must be suppressed to the inconspicuous level, therefore, further discussion must be implemented for the future.

\section{LC LENS}

LC lens can change the lens power with the control of applied voltages, and expect to apply for the unique tunable devices like auto focus function for micro camera with no moving parts. However, conventional LC lens can only increase the thickness of the LC layer in order to obtain the large retardation for advanced lens power or enlarge the lens aperture, practical applications of that were rather restricted with the same manner as discussed about LD deflector. Therefore, Fresnel type of LC lens was also designed with the multiple interdigitated electrodes aligned concentrically with the different pitches. That can make saw-tooth potential distribution with only planar surface of the transparent substrate, then we have manufactured the large aperture LC lens to discuss the design and optical characteristics.

\subsection{Electrode Pattern}

Optical micrograph of transparent electrode pattern and the thin film structures are shown in Fig.5 (a) and (b). Two extraction electrodes were imposed across the concentric interdigitated electrodes in order to simplify the electrical wiring and the device manufacturing process. The basic dimension and the thickness with the transparent substrates and thin films including the electrodes are the same as the deflector sample. In order to supply voltages to the concentric ring electrodes, floating electrode wiring through the insulator layer was proposed and the effect of the haze suppression was also reported ${ }^{[3]}$. Our fabricated lens sample has circle ITO electrode at the center of the aperture and also the ring interdigitated electrodes, therefore, we tried that plural extraction electrodes were aligned on the same surface as ring electrodes to decrease the manufacturing cost. Insulator gaps of the high-resistivity layer described in Fig.1 were placed also around the extraction electrodes to suppress the disturbance of the wavefront. Figure 5 (b) shows the crossing part of the interdigitated electrode and the extraction electrode. The gray colored area indicates the insulator gap along the electrode and there's no high-resistivity layer of yellow colored. Figure 6 shows the observed interference fringes on the central part of LC lens including extraction electrode without insulator gap (a) and with insulator gap (b). This structure can suppress the effect of the extraction electrode on the same surface and the concentric potential distribution was obtained as shown in Fig.6 (b). Patterning process of the electrodes with this alignment is not so complicated, therefore, that make the manufacturing process to be more simplified and may acquire the further cost merit at the mass production.
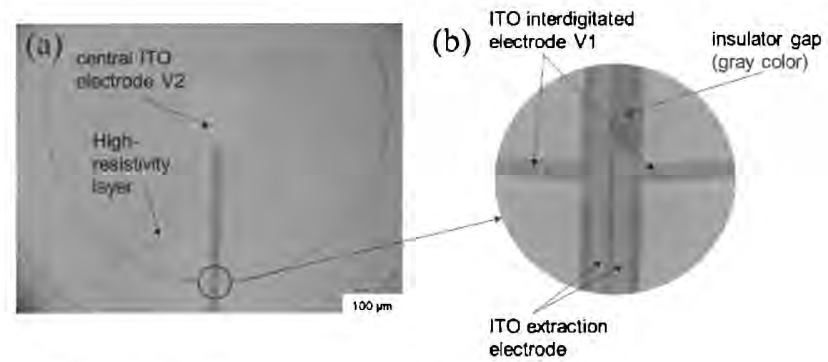

Figure 5. Optical micrograph of LC lens device.

(a) Central area of LC lens.

(b) Cross-point of extraction electrode of LC lens. (a)

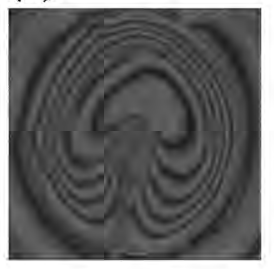

(b)

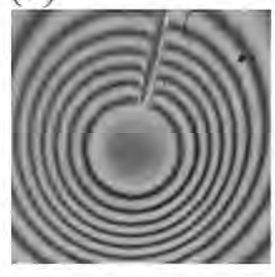

Figure 6. Optical micrograph of interference fringes.

(a) Extraction electrode without insulator gap.

(b) Extraction electrode with insulator gap. 


\subsection{Large Aperture Lens}

We have tried to enlarge the aperture of LC lens with the use of new Fresnel structure. The advanced transparent interdigitated electrodes were concentrically aligned on the lens aperture with the insulator gaps under their boundary area, we could obtain the concentric saw-tooth distribution of electrical potential in the liquid crystal layer; similar to the cross section of conventional Fresnel lens. The insulator gap placed along the extraction electrodes, as shown in 4.1, could reduce the disturbance of the modulated optical wavefront from the discontinuous electrode pattern. The radius of the $n_{\mathrm{th}}$ boundary line of the interdigitated electrodes was set to $R_{c} \mathrm{X} \mathrm{n}^{1 / 2}$ in order to equalize the retardation obtained in each concentric zone under the same applied voltages ${ }^{[7]}$. $R_{c}$ indicates the radius of the 1 st boundary line. In this study, we set the value of $R_{c}$ to $1.5 \mathrm{~mm}$. Therefore, segment pitches decreased linearly from the inner to the outer area. The diameter of the manufactured lens aperture was $30 \mathrm{~mm}$ and the number of the Fresnel zones was 100.

(a)

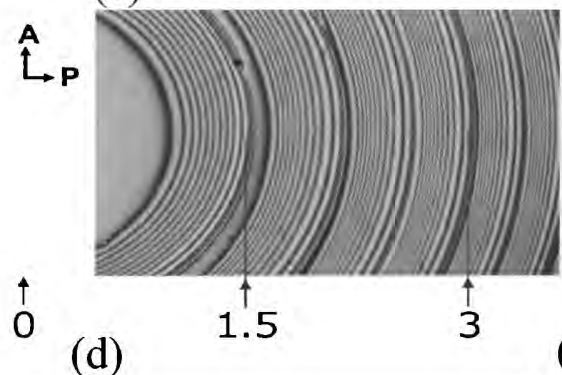

(b)

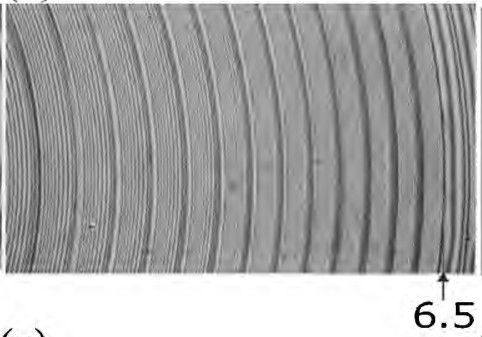

(e)

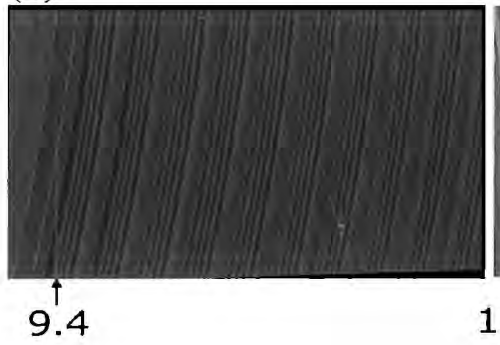

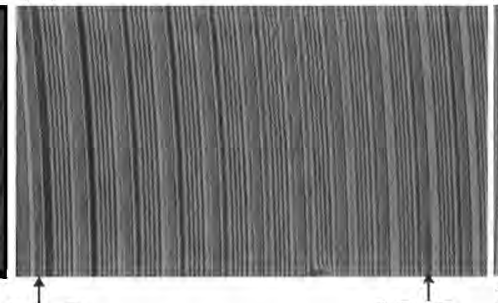

11.8 (c)

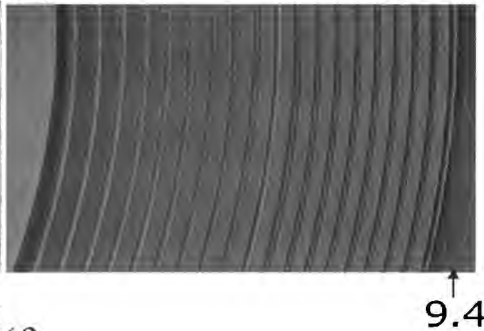

(f)

9.4

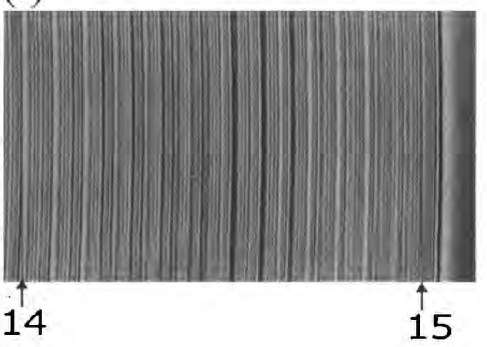

Lens Radius [mm]

Figure 7. Observed interference fringes in inner and outer area. Applied voltages $\left(V_{1}, V_{2}\right)$ are $(2.8,0.35)$ [ $\left.\mathrm{V}_{\mathrm{rms}}\right]$. Applied frequencies are as follows; (a), $20 \mathrm{kHz}$ (b), $20 \mathrm{kHz}$ (c), $20 \mathrm{kHz}$ (d), $50 \mathrm{kHz}$ (e), $200 \mathrm{kHz}$ (f), $200 \mathrm{kHz}$

Figure 7 (a) (f) shows the observed interference fringes from the polarizing microscope images. In this case, applied voltage was fixed but frequencies had to be changed from $20 \mathrm{kHz}$ to $200 \mathrm{kHz}$ to obtain the expected number of fringes. Fig. 8 shows the calculated phase retardation from each radial position of the interference fringe in Fig.7. The upward convex curve indicates the positive lens characteristics and the downward convex curve indicates the negative one. Equations of the fitting with 2nd degree polynomial approximation are also described for positive and negative lenses that gave the largest curvature. The square values of correlation coefficients indicate over 0.993 in both cases. The calculated lens powers from the equations are $3.97 \mathrm{~m}^{-1}$ and $-2.25 \mathrm{~m}^{-1}$, respectively in the large aperture of $30 \mathrm{~mm}$. The negative value of lens power indicates the negative (i.e. concave) lens function.

In our previous study, we evaluated the lens characteristics only inner portion with the aperture of $13 \mathrm{~mm}{ }^{[7]}$. In that case, the frequency of the applied voltage was fixed to $20 \mathrm{kHz}$ and the polynomial approximation was performed with the same manner. Obtained lens powers were found to be increased with the applied voltages in both polarities of convex and concave lens functions. The obtained square values of correlation coefficients were indicated over 0.9995 in both polarities and the calculated lens powers from the equations were $3.97 \mathrm{~m}^{-1}$ and $-4.67 \mathrm{~m}^{-1}$, respectively in the aperture diameter of $13 \mathrm{~mm}$. Because LC lens was designed with different segment pitches of the interdigitated electrodes, the desirable number of the interference fringes will be almost the same as described above. However, the obtained number 
of the fringes in the outside zone was $8 \sim 7$ as shown in Fig.7 (f) ; a little smaller than that in the inside zone, $11 \sim 10$ as shown in Fig.7 (a),(b). This reduction of fringes in the outer area is more remarkable in the case of concave lens. That's why the obtained negative lens power was not enough while positive one is enough, as shown in Fig.8. This mismatch of the number was found to be occurred from the different optimal frequency of voltage application with each width of segment pitch. We would like to discuss about the matter in the next section.

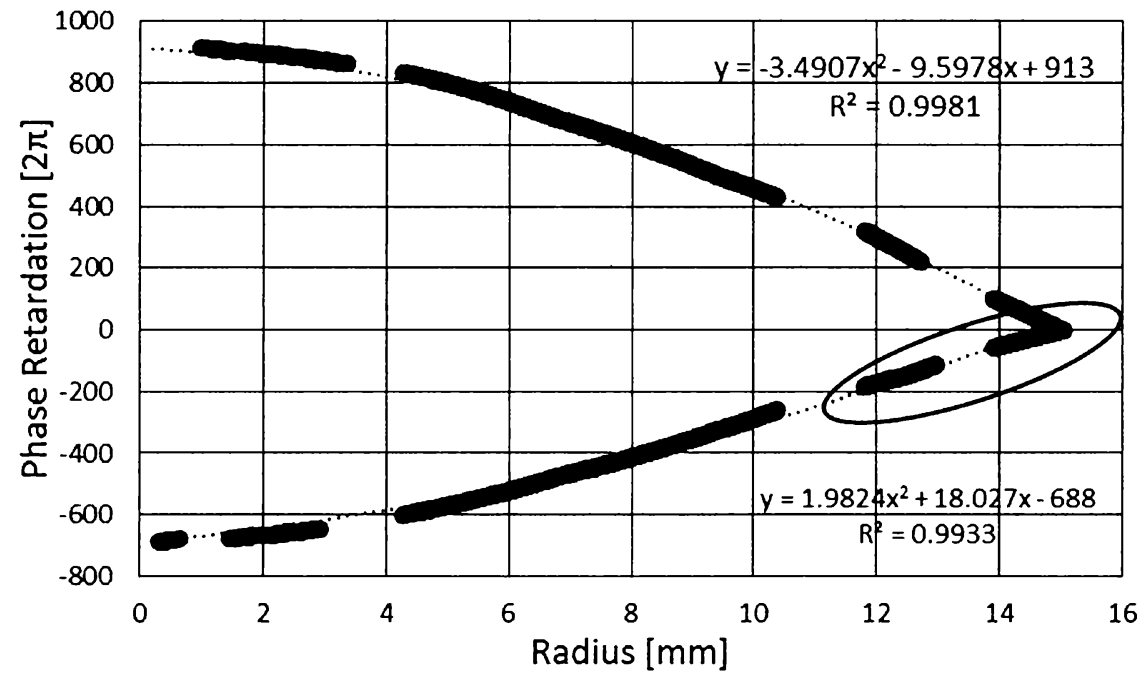

Figure 8. Phase retardations calculated from interference fringes.

\subsection{Dependence of optimal frequency on segment pitch}

When the LC lens is driven by alternative current voltage application, applied frequency is also important factor to control the lens characteristics ${ }^{[1]}$. In this study, when the interdigitated electrode was placed, there found to be a strong relationship between a segment pitch and an applied frequency. In particular, segment pitch of the LC lens decreases from the inner to the outer area, therefore we have to consider the optimal driving frequency on the all area of the lens radius. Then we have discussed the potential level obtained from the concentric interdigitated segment with a finite element analysis software (COMSOL Inc., COMSOL Multiphysics) ${ }^{[1][6]}$.

Figure 9 shows the calculated potential distributions from the inner to the outer area of the LC lens aperture with the same manner as described in Fig.1 (b). Used model was assumed an intersection of circular LC lens, so segment pitch is decreased from the inner to the outer area. Therefore, be careful to the $\mathrm{X}$-axis which indicates the radial position of the lens described with the different scale. Figure 9 (a) (c) show the calculation result of potential distribution driving with the same frequency of $200 \mathrm{~Hz}$. In this condition, amplitude of the obtained potential shows shrink with the segment pitch. Figure 9 (d) (f) show the potential distribution driving with the variant frequency as indicated. This condition, we can obtain the sufficient amplitude of the potential both the inner and the outer area. According to the results, amplitude of the potential in the LC material was found to be shrunk with the decreasing of segment pitch. On the other hand, voltage application with higher frequency seems to recover the shrunk amplitude to be larger. That's why we had to change the frequency of applied voltage to be higher on the outer lens area than inner area to obtain the sufficient lens power, we supposed. According to our further discussions, this difference of the optimal frequency was found to be caused by the higher impedance of the insulator layer of vertical direction. Therefore, our next challenge is to reduce the difference of the optimal frequency between the inner and the outer area, otherwise, we have to prepare the voltage source of multi frequencies which causes the increasing of the driving cost. When we develop an advanced LC lens with larger aperture, this problem must be overcome as well as increasing the lens performance. One solution is to improve 
the device structure to be able to apply different frequency independently for each area. Another approach is to reduce the dependence of driving frequency on the segment pitch itself. If possible, the latter solution is better from the purpose to simplify the device structure and saving the driving cost.
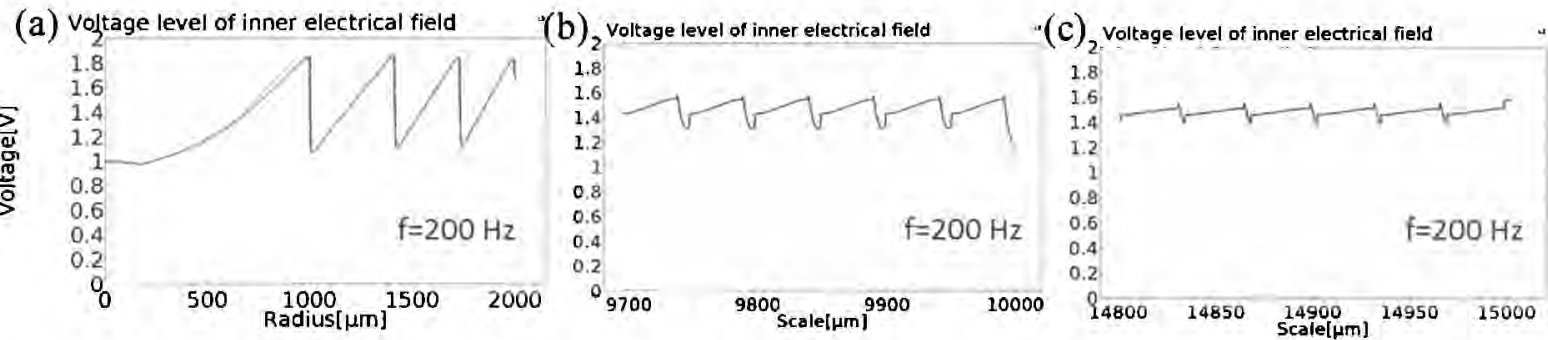

(d) Voltage level of inner electrical field

(e) Volztage level of inner electrical field
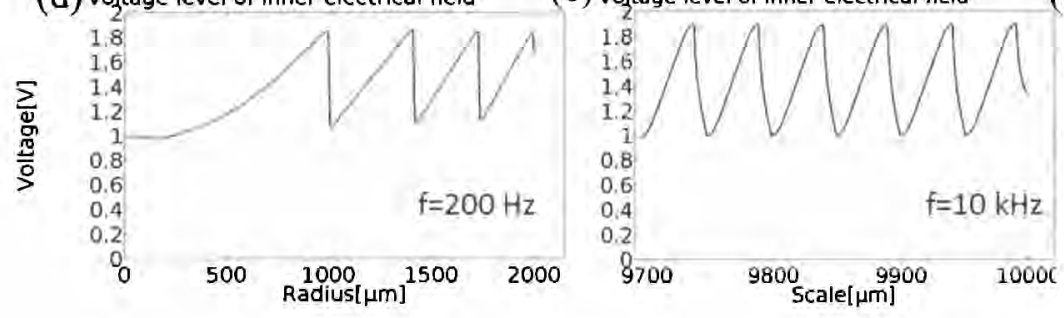

(f) Voltage level of inner electrical field

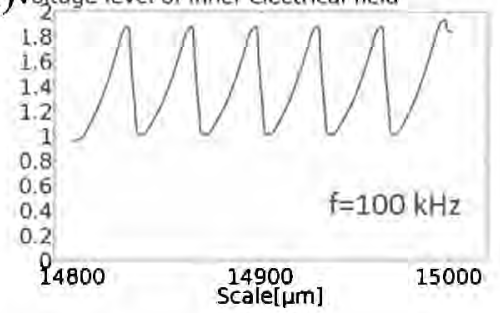

Figure 9. Calculated potential distributions from inner to outer area of the LC lens aperture.

Applied voltages $\left(V_{l}, V_{2}\right)$ are $(1.0,2.0)$ [ $\left.\mathrm{V}_{\mathrm{rms}}\right]$.

Applied frequencies are as follows; (a), $200 \mathrm{~Hz}$ (b), $200 \mathrm{~Hz}$ (c), $200 \mathrm{~Hz}$ (d), $200 \mathrm{~Hz}$ (e), $10 \mathrm{kHz}$ (f), $100 \mathrm{kHz}$

\section{CONCLUSION}

Tunable optical LC devices with Fresnel structure was designed, manufactured and evaluated. Advanced LC deflector with interdigitated electrodes aligned into $200 \mu \mathrm{m}$ segment shows the improvement of beam deflection angle of \pm 1.3 deg. with no moving parts. Captured image could be moved under the beam deflection control by LC deflector and supposed to be possible to apply for the OIS function of micro camera. However, next technical target was also clarified that haze of the captured image must be suppressed during the beam deflection. $\mathrm{LC}$ lens with large aperture of $30 \mathrm{~mm}$ diameters was achieved with Fresnel structure and obtained tunable range was estimated $\pm 4\left[\mathrm{~m}^{-1}\right]$ with the LC thickness of $40 \mu \mathrm{m}$. The extraction electrode through the concentric interdigitated electrode works well to avoid the disturbance of optical wavefront by the guarded insulator gap around. The next challenge is found to decrease the difference of optimal driving frequency between the segment in the inner area and the outer area. Obtained experimental results suggest their present tasks of the Fresnel type devices; reduction of diffraction light and the countermeasure against the dependence of optimal frequency on the different segment pitch. Then we are going to evaluate and improve the dynamic performance of the devices into the practical level, and promote the extraction and the resolution of problems and also try to create the new killer applications of LC devices.

\section{ACKNOWLEDGEMENT}

This work was supported by the Photonics Center, Osaka University, under the program of MEXT Photonics Advanced Research Center Program. Our research and development was also adopted by "Project for Creating STart-ups from Advanced Research and Technology" (START), 2016, JST. We are very grateful to their generous support. 


\section{REFERENCES}

[1] Giichi Shibuya, Hiroyuki Yoshida and Masanori Ozaki, "High speed driving of liquid crystal lens with weakly conductive thin films and voltage booster," Applied Optics Vol.54 (27), 8145-8151 (2015)

[2] Mao Ye, Bin Wang, Masaru Uchida, Satoshi Yanase, Shingo Takahashi and Susumu Sato, "Focus tuning by liquid crystal lens in imaging system," Applied Optics Vol.51, No.31,7630-7635 (2012)

[3] Liwei Li, Doug Bryant, Tony Van Heugten and Philip J. Bos,"Near-diffraction-limited and low-haze electrooptical tunable liquid crystal lens with floating electrodes," Optics Express Vol.21, No.7, 8371-8381 (2013)

[4] Hung-Chun Lin and Yi-Hsin Lin, "A fast response and large electrically tunable-focusing imaging system based on switching of two modes of a liquid crystal lens," Applied Physics Letters 97, 063505 (2010)

[5] $\mathrm{Su} \mathrm{Xu,} \mathrm{Yan} \mathrm{Li,} \mathrm{Yifan} \mathrm{Liu,} \mathrm{Jie} \mathrm{Sun,} \mathrm{Hongwen} \mathrm{Ren} \mathrm{and} \mathrm{Shin-Tson} \mathrm{Wu,} \mathrm{"Fast-response} \mathrm{liquid} \mathrm{crystal} \mathrm{microlens,"}$ Micromachines 2014, 5, 300-324 (2014)

[6] Giichi Shibuya, Hiroyuki Yoshida and Masanori Ozaki, "Tunable optical deflector with Fresnel type of liquid crystal device," IDW'15 LCTp6-4, 170-173 (2015)

[7] Giichi Shibuya, Shohei Yamano, Hiroyuki Yoshida and Masanori Ozaki, "Tunable Optical Liquid Crystal Deflector and Lens with Fresnel Structure," IDW/AD'16 LCT5-3, 106-109 (2016)

[8] Hongwen Ren, Yun-Hsing Fan and Shin-Tson Wu, "Prism grating using polymer stabilized nematic liquid crystal," Applied Physics Letters 82 (19), 3168-3170 (2003)

[9] Roy M. Matic, "Blazed phase liquid crystal beam steering," Proceedings of SPIE 2120, 194-205 (1994)

[10] Werner Klaus, Masafumi Ide, Shigeru Morokawa, Masahiro Tsuchiya and Takeshi Kamiya, "Angle-independent beam steering using a liquid crystal grating with multi-resistive electrodes," Optics Communications 138, 151-157 (1997)

[11] Katsuhiko Hirabayashi, Tsuyoshi Yamamoto and Masayasu Yamaguchi, "Free-space optical interconnections with liquidcrystal microprism arrays," Applied Optics Vo.34, No.14, 2571-2580 (1995) 